\title{
Pathogenesis, Diagnosis and Treatment of Chronic Lymphocytic Leukemia: Exciting Times
}

\author{
Michael Hallek \\ Department I of Internal Medicine, University Hospital of Cologne, Cologne, Germany
}

The management of chronic lymphocytic leukemia (CLL) is currently undergoing profound changes. Therefore, the editors of Oncology Research and Treatment have decided to publish a minireview series on this dynamic field.

CLL is the commonest leukemia in Western countries. The disease typically occurs in elderly patients and has a highly variable clinical course. Leukemic transformation is initiated by specific genomic alterations that impair apoptosis of clonal B cells. Frenzel et al. [1] summarize the essential pathogenic events in CLL in their article 'Concepts of CLL pathogenesis: DNA damage response and tumor microenvironment'.

Two prognostic staging systems exist, the Rai and Binet staging systems, which are established by physical examination and blood counts. In addition, various biological and genetic markers also have prognostic value. For example, deletions of the short arm of chromosome 17 (del(17p)) predict resistance to available chemotherapies; usually a poorer outcome with a shorter time to progression or survival. In their article 'Genomic features: impact on pathogenesis and treatment', Tausch et al. [2] summarize the exciting developments of genomic discoveries in CLL and give guidance which markers or genes should be used in general practice in 2016 .

While it is consensus that only patients with active or symptomatic disease or with advanced Binet or Rai stages require therapy, it has been an important scientific question for a long time, whether early therapy could possibly alter and improve the natural course of this leukemia. In their article 'Early treatment in CLL: what is the current evidence?' Langerbeins et al. [3] address the current status on this important question.
In the final paper, Cramer et al. [4] 'State of the art treatment and novel agents in CLL' give an overview on the current treatment options in CLL, in light of the many new drugs that have been approved recently or are about to be approved. For physically fit patients, chemoimmunotherapy with fludarabine, cyclophosphamide, and rituximab remains the current standard therapy. For unfit patients, treatment with an anti-CD20 antibody (obinutuzumab, rituximab, or ofatumumab) plus a milder chemotherapy (chlorambucil) may be applied. Several new agents (e.g., ibrutinib, idelalisib, obinutuzumab) hold the potential to improve the outcome of CLL patients. However, their optimal use (in terms of combination, sequence, and duration) is unknown. Therefore, CLL patients should be treated in clinical trials testing these new agents whenever possible (for more information visit www.dcllsg.de). In our new section 'Ongoing Clinical Trials' (see page 69) Kirsten Fischer describes the CLL14 trial that will test combinations of new agents (obinutuzumab, venetoclax) in CLL. This trial might lead the way to chemotherapy-free treatments of CLL.

\section{Disclosure Statement}

The author received research funding and honoraria for scientific talks and advisory boards from the following pharmaceutical companies: AbbVie, Celgene, Gilead, GlaxoSmithKline/ Novartis, F. Hoffmann LaRoche, JanssenCilag/Pharmacyclics and Mundipharma.

\section{References}

1 Frenzel LC, Reinhardt HC, Pallasch CP: Concepts of CLL pathogenesis: DNA damage response and tumor microenvironment. Oncol Res Treat 2016;39: DOI: $10.1159 / 000443820$.

2 Tausch E, Mertens D, Stilgenbauer S: Genomic features: impact on pathogenesis and treatment of chronic lymphocytic leukemia. Oncol Res Treat 2016;39: DOI: $10.1159 / 000443906$.
3 Langerbeins P, Groß-Ophoff-Müller C, Herling CD: Risk-adapted therapy in early-stage chronic lymphocytic leukemia. Oncol Res Treat 2016;39: DOI:10.1159/ 000443013.
4 Cramer P, Hallek M, Eichhorst B: State of the art treatment and novel agents in chronic lymphocytic leukemia. Oncol Res Treat 2016;39: DOI: 10.1159/000443903.

\section{KARGER}

Fax +497614520714

\section{() 2016 S. Karger GmbH, Freiburg}

2296-5270/16/0391-0008\$39.50/0
Prof. Dr. med. Michael Hallek

Department I of Internal Medicine

University Hospital of Cologne

Joseph-Stelzmann-Straße 9, 50924 Cologne, Germany

michael.hallek@uni-koeln.de 